\title{
Photoacoustic and photothermal beam deflection as a probe of laser ablation of materials
}

\author{
Jeffrey A. Sell and David M. Heffelfinger \\ Physics Department, General Motors Research Laboratories, Warren, Michigan 48090-9055 \\ Peter L. G. Ventzek and Ronald M. Gilgenbach \\ Intense Energy Beam Interaction Laboratory, Nuclear Engineering Department, University of Michigan, \\ Ann Arbor, Michigan 48109-2104
}

(Received 20 August 1990; accepted for publication 16 October 1990)

\begin{abstract}
Photoacoustic and photothermal laser-beam deflection were applied as diagnostics of the pulsed ultraviolet (UV) laser ablation of a polymer polyethyleneterephthalate. Here, a continuous-wave (cw) laser beam is passed parallel to the sample, but displaced from it by a few hundred micrometers. A density gradient caused by the pulsed UV laser heating or ablation of the sample deflects the cw laser beam. This deflection is measured directly using a position-sensitive detector. A quantitative model of the photothermal deflection at low fluence was developed which fits the data very well. This enabled a new method of measuring the thermal diffusivity of the fluid in contact with the sample. Distortion of the photothermal and photoacoustic signal as the excimer fluence is raised through the ablation threshold allowed the determination of the threshold. Also, the velocity of the ablation products was measured through a time-of-flight analysis and found to be dependent on the laser fluence used, the nature of the gas above the sample, and the distance above the sample at which the velocity is measured. The beam deflection in a vacuum is used to measure the ablation product velocity.
\end{abstract}

\section{INTRODUCTION}

We report here the application of photothermal and photoacoustic laser-beam deflection to the problem of pulsed UV (excimer) laser ablation of materials. Preliminary accounts of our work have appeared previously. ${ }^{1-4}$ The results in the present paper were obtained using excimer laser $\mathrm{KrF}(248 \mathrm{~nm})$ ablation of a polymer polyethyleneterephthalate (PET). However, our previous paper ${ }^{1}$ showed the applicability of the method to a wide variety of materials.

In this experiment a beam from a low-power cw laser beam such as a $\mathrm{He}-\mathrm{Ne}$ is passed parallel to the substrate surface, but displaced from it by typically a few hundred micrometers to a few millimeters. A position-sensitive detector, located roughly $1 / 4 \mathrm{~m}$ away, measures the deflection angle of the probe beam due to index of refraction gradients in the gas above the sample from the pulsed UV laser incident normally to it. This is commonly called photothermal deflection (PD) or mirage detection.

Previous applications of photothermal deflection are numerous and include spectroscopy, ${ }^{5-9}$ velocimetry, ${ }^{10-12}$ transport and relaxation, ${ }^{13}$ analytical detection, ${ }^{14}$ desorption, ${ }^{15}$ and measurements of diffusivity. ${ }^{16,17}$ Similar applications have been previously demonstrated for photoacoustic beam deflection. ${ }^{18-24}$ Wetsel, Stotts, and Clark ${ }^{25}$ have generated photoelastic waves in materials using a pulsed laser and detected them using probe-beam deflection. Jamieson and Wetsel ${ }^{26}$ have studied blast waves above nonablating solid sample surfaces using optical beam deflection and found the blast wave speed to be dependent on the heating laser-pulse energy.

Thus, this method is not new, but the full extent of the information furnished by the technique in the application involving pulsed-laser photoablation has not yet been described. Our work is an extension of that done previously by Koren, ${ }^{27}$ Petzoldt and co-workers, ${ }^{28,29}$ Enloe, Gilgenbach, and Meachum, ${ }^{30,31}$ and Dreyfus, McDonald, and von Gutfield. ${ }^{32,33}$ Koren ${ }^{27}$ used probe-beam deflection above the sample to observe shock waves and cooling waves. However, the discrete detector used did not allow interpretation of the data in terms of a deflection angle, and he did not use the method to identify the ablation threshold. Petzoldt and co-workers ${ }^{28,29}$ used probe-beam deflection to measure the damage threshold from a pulsed visible dye laser on optical materials. They measured the acoustic pulse energy as a function of the incident-beam intensity. The present work also deals with the distortion of the acoustic and thermal deflection signal as the laser fluence is raised through the ablation threshold. Our earlier report ${ }^{3}$ showed that this distortion is due to a transition from a sound wave to a shock wave which occurs at the onset of ablation. Enloe and co-workers ${ }^{30,31}$ analyzed probe-beam deflection as a result of plasma formation from a pulsed laser incident on a polymer surface. Material ablation may or may not be accompanied by plasma formation, but the technique can still be used in the absence of a plasma as shown here. Dreyfus and co-workers ${ }^{32,33}$ used probe-beam deflection from a surface deformation to examine energy deposition below the ablation threshold.

\section{EXPERIMENT}

The experimental apparatus is shown in Fig. 1. The ablation source was a focused beam of pulsed UV radiation from a $\mathrm{KrF}$ excimer laser operating at $248-\mathrm{nm}$ wavelength 


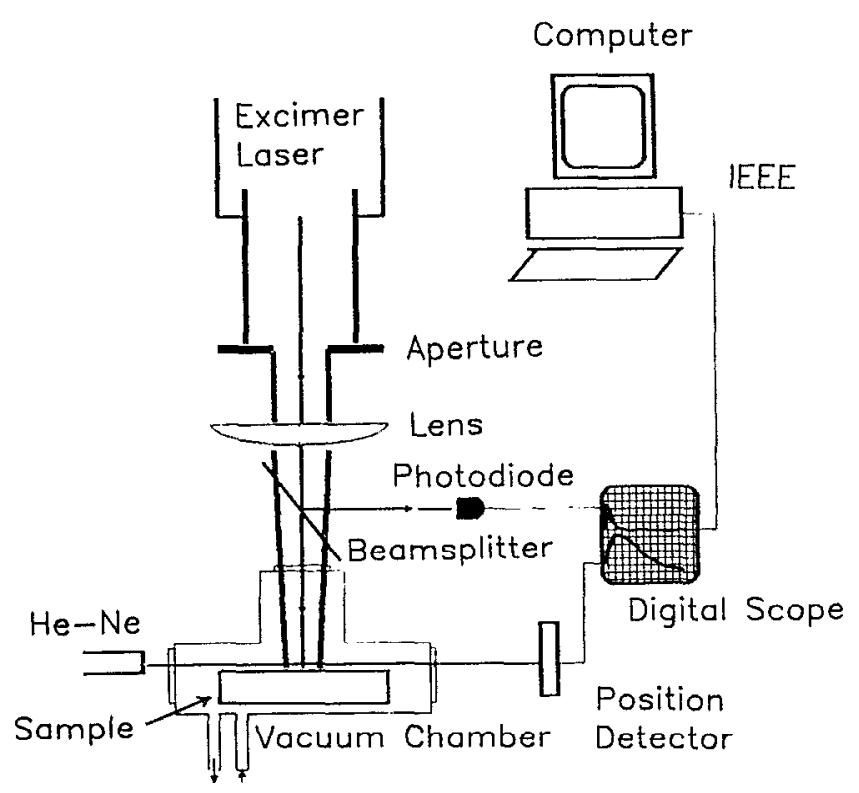

FIG. 1. Diagram of the experiment.

with a maximum energy of $300 \mathrm{~mJ} /$ pulse and a repetition rate of $0.1 \mathrm{~Hz}$. This slow repetition rate was necessary to avoid sample heat accumulation. The laser was enclosed in a Faraday cage to reduce $\mathrm{rf}$ emission; this was particularly important for high-speed data acquisition.

The excimer beam was apertured by a rectangular slit for best beam uniformity and focused by a cylindrical lens to a rectangular spot. The width of the spot was much greater than the diameter of the probe beam. A probe beam, a $\mathrm{He}-\mathrm{Ne} \mathrm{cw}$ laser of $4 \mathrm{~mW}$ power focused by a lens $(f=125 \mathrm{~mm})$ to a waist of 80 micrometers, passed parallel to the sample surface oriented normally to the excimer beam and parallel to the long axis of the excimer rectangular spot. The Rayleigh range of the focused $\mathrm{He}-\mathrm{Ne}$ beam was found to be roughly $3 \mathrm{~cm}$, twice the length of the excimer rectangular spot.

A range of fluences incident on the sample surface was achieved by varying the laser energy per pulse, by attenuating the laser energy with quartz plates $(\approx 10 \%$ attenuation per plate), and by changing the distance between the pump focusing lens and the sample surface (hence, varying the pump-laser spot size). The spot size of the excimer beam was measured from light-sensitive paper. The error bars in the fluence values are estimated to be less than $10 \%$. In order to make single-shot measurements, a calibrated photodiode was used to monitor the energy per pulse of the excimer pump laser.

The samples were mounted in a vacuum chamber which was a six-way cross pumped by a turbomolecular pump typically to $1 \times 10^{-5}$ Torr. The chamber was mounted on an $X-Y-Z$ translation stage. A translation vernier determined the $z$ distance, which is the distance between the sample surface and probe-beam center.

The deflection signal was obtained in a vacuum as well as in dry air at $1 \mathrm{~atm}$. Some experiments were also per-

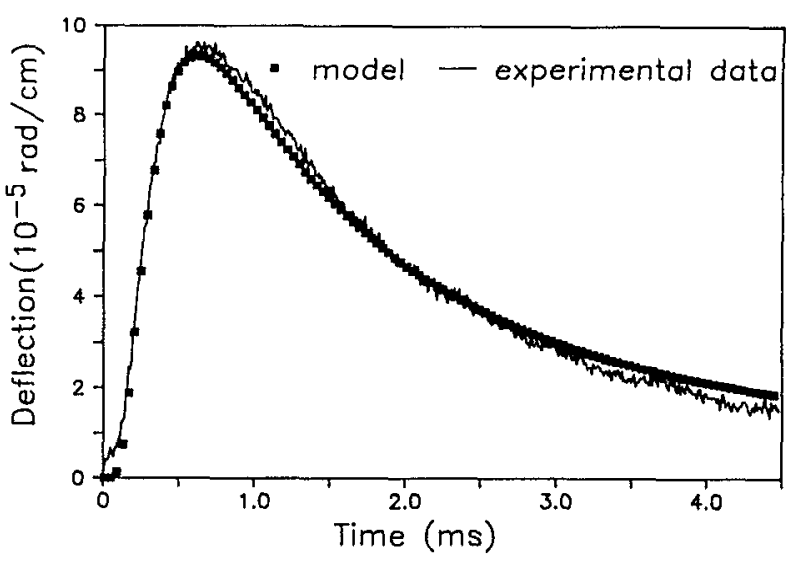

FIG. 2. Photothermal deflection signal (average of four shots) for PET obtained in air with a laser fluence $17 \mathrm{~mJ} / \mathrm{cm}^{2}$ and a probe beam height of $280 \mu \mathrm{m}$. The model data were multiplied by a factor of 0.55 for ease of comparison with the experimental data. For PET the diffusivity is $D=7.8 \times 10^{-4} \mathrm{~cm}^{2} / \mathrm{s}$ and the thermal conductivity is $k=1.4 \times 10^{-3}$ $\mathrm{W} /(\mathrm{cm} \mathrm{K})$.

formed in dry air at various pressures and in $\mathrm{He}, \mathrm{Xe}$, and $\mathrm{N}_{2}$ at 1 atm.

Deflection of the probe beam was measured by a quadrant cell photodiode, which allows direct calculation of the probe-beam deflection angle. The sensitivity of the position sensor was $702 \mathrm{~V} / \mathrm{rad}$, and the response time was characterized previously ${ }^{30}$ as $20 \mathrm{~ns}$. Data from the position sensor and photodiode energy measurement were collected by a two-channel Tektronix digital oscilloscope and stored via an IEEE interface on a computer. In addition, the deflection signals were simultaneously recorded on an oscilloscope $(75 \mathrm{MHz})$ where photographs of the traces were taken. All data discussed here are single shot unless otherwise specified. The deflection signals shown here are given in units of rad $/ \mathrm{cm}$ where $\mathrm{cm}$ refers to the path length of the overlap of the probe beam with the excimer beam. This was done since different excimer-beam spot sizes were used to vary the fluence; the signal was normalized by path length since the signal depends linearly on it. However, for the plots shown, the path length was always $1.65 \mathrm{~cm}$ so that conversion to radians is accomplished by multiplying the data by 1.65 .

All samples studied here were "aged" by applying 100 pulses of relatively low fluence before actually taking any data. The intent here was to desorb any surface contaminants, especially water vapor.

\section{RESULTS AND DISCUSSION}

\section{A. Photothermal beam deflection in a gaseous atmosphere}

We begin discussion of the deflection results with the polymer PET. Figure 2 shows the probe-beam deflection signal at low fluence $\left(17 \mathrm{~mJ} / \mathrm{cm}^{2}\right)$ in air which appears as a rapid rise followed by a slow decay. As shown below, this signal can be accounted for entirely as a thermal deflection signal. The UV pulse from the excimer laser simply heats the polymer surface, and this heat subsequently conducts 
into the adjacent air. The probe beam is deflected due to the time-dependent density (and therefore index of refraction) gradient that diffuses through the probe-beam path. We call this the photothermal deflection signal as opposed to a photoacoustic deflection signal which is observed at earlier times and to be discussed below. Figure 2 also shows a deflection signal calculated by the thermal model discussed next.

In order to calculate the deflection angle $\phi$, it is first necessary ${ }^{5}$ to compute the temperature gradient of the index of refraction $n$ :

$$
\phi=\frac{1}{n} \int d s \nabla_{\perp} n .
$$

For our experiment this simplifies to

$$
\phi=\frac{1}{n} \frac{\partial n}{\partial T} \frac{\partial T}{\partial z} L,
$$

where $L$ is the overlap length of the pump and probe beams, and $T$ is the temperature.

Since $\partial n / \partial T$ is essentially a constant (for a fluid that remains close to ambient temperature), then what remains is to calculate the time-dependent temperature $T$ for the fluid above the substrate heated on the surface by a laser pulse. According to Ref. 34, the expression for $T_{g}$, the temperature of the gas above the sample at time $t$, and distance $z$ above the surface, is

$$
T_{g}=\frac{k_{s} D_{g} D_{s}^{-1 / 2}}{\left(k_{s} \sqrt{D_{g}}+k_{g} \sqrt{D_{s}}\right) \sqrt{\pi D_{g} t}} e^{-z^{2} / 4 D_{g^{t}}}
$$

where $k_{g}$ and $D_{g}$ are the thermal conductivity and thermal diffusivity of the gas, respectively, and $k_{s}$ and $D_{s}$ are those for the solid, obtained with the approximation that the laser beam is completely absorbed on the surface, the laser is an instantaneous unit planar source, and there is no contact resistance at the solid-gas boundary. Differentiating Eq. (3), then substituting into Eq. (2), introducing the laser energy density $E_{0}$ and simplifying leads to

$$
\begin{aligned}
\phi= & \frac{1}{n} \frac{\partial n}{\partial T} \frac{L E_{0}}{2 \sqrt{\pi} \rho_{g} C_{g}} \sqrt{\frac{D_{s}}{D_{g}}} \frac{z}{\left(D_{g} t\right)^{3 / 2}} \\
& \times e^{-z^{2} / 4 D_{g^{t}}(1+\Gamma) \frac{k_{g} / k_{s}}{2}},
\end{aligned}
$$

where $\Gamma$ is the thermal mismatch defined as

$$
\Gamma=\frac{\left(e_{s}-e_{g}\right)}{\left(e_{s}+e_{g}\right)}
$$

where $e_{s}$ and $e_{g}$ are the effusivities of the solid and gas, respectively. Here,

$$
e_{s}=\sqrt{k_{s} \rho_{s} C_{s}}
$$

and likewise for the gas. Also, $\rho_{g}$ and $C_{g}$ are the density and specific heat of the gas, respectively.

Noting that $\phi$ reaches a maximum when

$$
\frac{\partial \phi}{\partial t}=0 \text {, }
$$

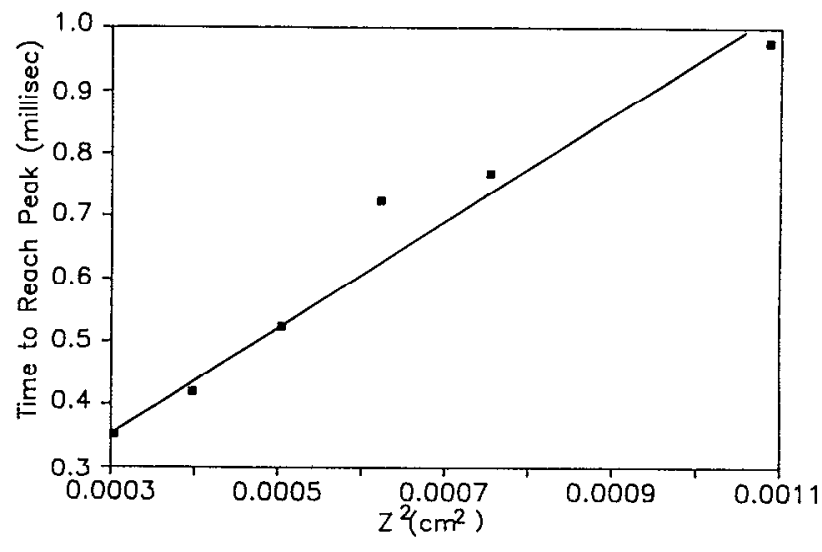

FIG. 3. Plot of the time for the PD signal to reach a peak value vs $z^{2}$, where $z$ is the distance of the center of the probe beam to the sample surface. Fluence was $12 \mathrm{~mJ} / \mathrm{cm}^{2}$.

we find that the time at which the maximum is reached $t_{\max }$ is

$$
t_{\max }=\frac{z^{2}}{6 D_{g}} .
$$

Thus a plot of $t_{\max }$ vs $z^{2}$ should be a straight line whose slope is $1 / 6 D_{g}$, yielding the value for $D_{g}$. Such a plot is shown in Fig. 3 for PET using a fluence of $12 \mathrm{~mJ} / \mathrm{cm}^{2}$. The slope yields a value of $D_{g}=0.20 \mathrm{~cm}^{2} / \mathrm{s}$, in good agreement with table values of $D_{g}(0.21)$ for air at room temperature and pressure. Substituting (8) into (4) yields

$$
\phi_{\max }=6^{3 / 2} e^{-3 / 2} c\left(\frac{1}{z^{2}}\right)
$$

where $c$ is a constant. We have also verified the $1 / z^{2}$ dependence of $\phi_{\max }$ (data not shown here).

It is interesting to consider the pressure dependence of Eq. (8). Since the diffusivity $D_{g}$ is inversely dependent on pressure,

$$
D_{g}=D_{g}^{0} \frac{P^{0}}{P},
$$

then the time to reach peak pressure $t_{\max }$ should depend linearly on pressure:

$$
t_{\max }=\frac{z^{2} P}{6 D_{g}^{0} P^{0}} .
$$

Experimental verification of this is shown in Fig. 4; the slope yields the same value for $D_{g}$, namely, $0.20 \mathrm{~cm}^{2} / \mathrm{s}$. We believe that these methods of measuring the thermal diffusivity have not been reported before, although Loulergue and Tam ${ }^{16}$ have previously reported measurements of the diffusivity of a doped, unconfined hot gas using a collinear beam-deflection technique. Also, similar measurements above surfaces have been reported ${ }^{17}$ using chopped $\mathrm{cw}$ radiation.

Note that if we insert the expression (8) into Eq. (4), and include the pressure dependence of the density, gas 


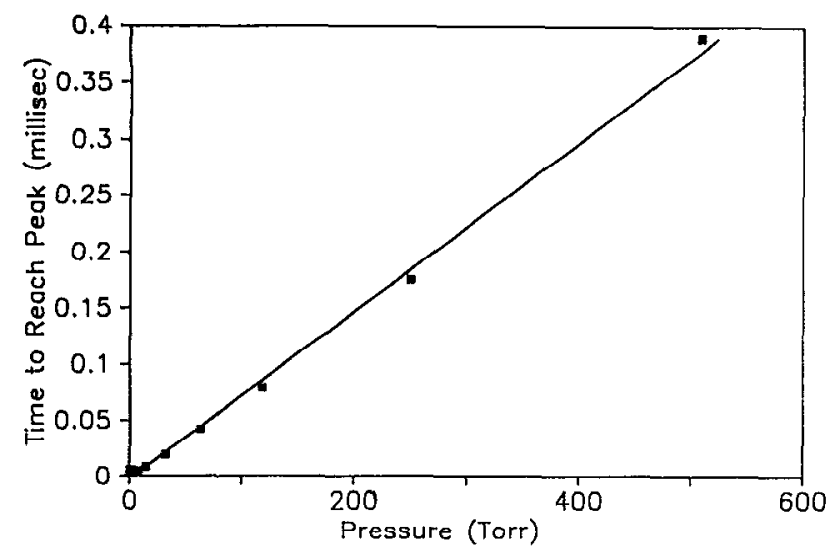

FIG. 4. Plot of time to reach peak deflection vs pressure for $z=264 \mu \mathrm{m}$ and a fluence of $18 \mathrm{~mJ} / \mathrm{cm}^{2}$.

diffusivity, and $\partial n / \partial T$, one can easily show that $\phi_{\max }$ depends on pressure $P$ as $\sqrt{P}$. We have measured this for PET and found it to hold quite well over a pressure range of 4-760 Torr, shown in Fig. 5.

If the heating of the solid induces a velocity of the gas $v$ in the $z$ direction, then we would expect, by analogy with Ref. 10, that the deflection angle $\phi$ is given by

$$
\begin{aligned}
\phi= & -\frac{1}{n} \frac{\partial n}{\partial T} \frac{L E_{0}}{2 \sqrt{\pi} \rho_{g} C_{g}} \sqrt{\frac{D_{s}}{D_{g}}} \frac{|v t-z|}{\left(D_{g} t\right)^{3 / 2}} \\
& \times e^{-(l t-z)^{2} / 4 D_{g^{t}}(1+\Gamma) \frac{k_{g} / k_{s}}{2} .}
\end{aligned}
$$

This expression is used to calculate the deflection angle $\phi$; it is compared to the experimental data in Fig. 2. The experimental values for the parameters used in the model are given in the caption. The agreement between the model and experiment is quite good.

Figure 6 shows the deflection of the probe beam in air

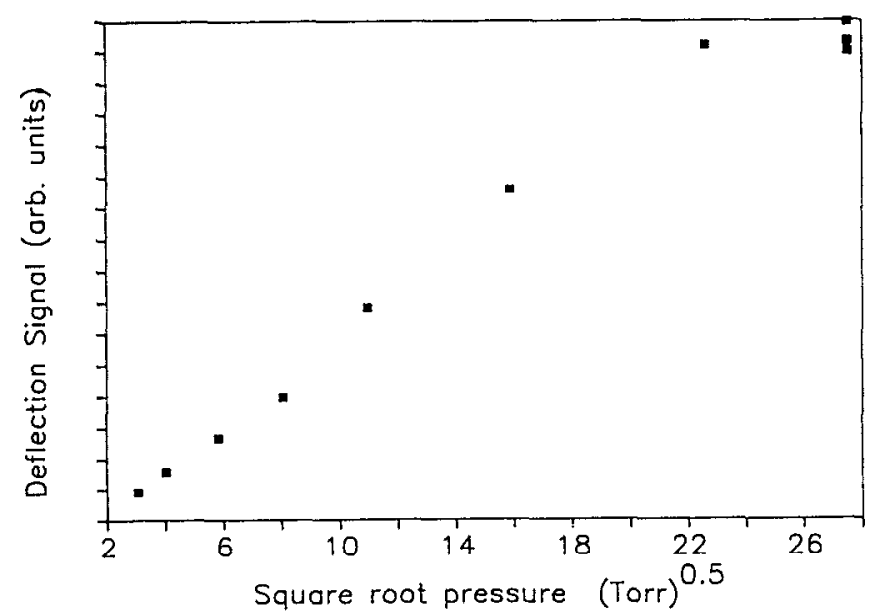

FIG. 5. Amplitude of photothermal deflection signal as a function of the square root of gas pressure for PET. Data obtained at a fluence of 18 $\mathrm{mJ} / \mathrm{cm}^{2}$ with a probe-beam height of $200 \mu \mathrm{m}$. The data points at the highest pressure are lower than expected because of a shift in the sample position.

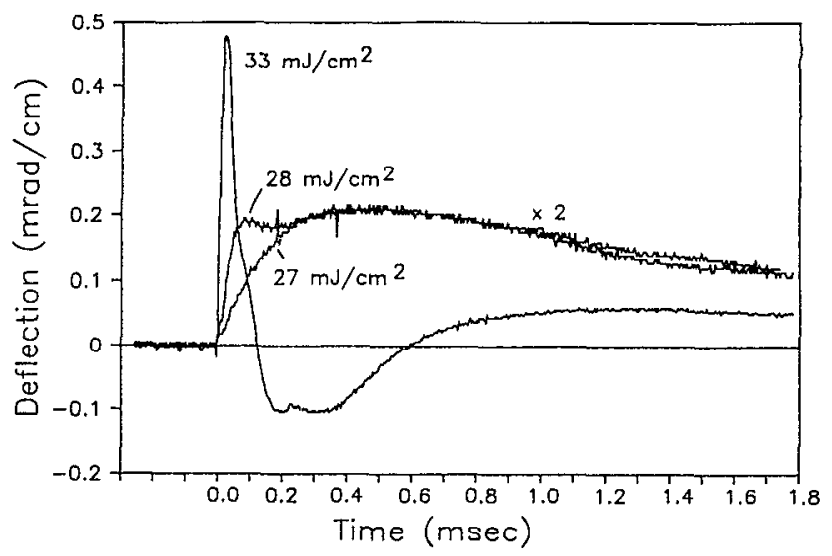

FIG. 6. Beam-deflection signal as a function of fluence for PET obtained in air at $1 \mathrm{~atm}$ at a probe-beam height of $225 \mu \mathrm{m}$.

for three different values of the excimer-laser fluence: 27 , 28 , and $33 \mathrm{~mJ} / \mathrm{cm}^{2}$. Below the ablation threshold, the deflection signal has no zero crossing (see the curves for 27 and $28 \mathrm{~mJ} / \mathrm{cm}^{2}$ ). Although not shown in the figure, we have verified that the deflection amplitude scales linearly with excimer fluence from 11 to $22 \mathrm{~mJ} / \mathrm{cm}^{2}$, indicating a constant fraction of energy deposition into the solid which conducts into the air. Above the threshold, the probe-beam deflection has a zero crossing (see the $33 \mathrm{~mJ} / \mathrm{cm}^{2}$ curve) and a large-amplitude, rapid component. We believe that the distorted shape of the deflection signal is due largely to the convective plume of the gaseous air/ablation product mixture. We show in Fig. 7 the computed beam-deflection signal using Eq. (12) for $v-0$ and $200 \mathrm{~cm} / \mathrm{s}$. The beamdeflection signal distorts much like the experimental data shown in Fig. 6. Although it may be possible to actually fit the beam-deflection data for fluences above the ablation threshold using Eq. (12) much like we did below the threshold in Fig. 2, we would not expect Eq. (12) to apply quantitatively for the ablating situation since it ignores index-of-refraction gradients produced by composition

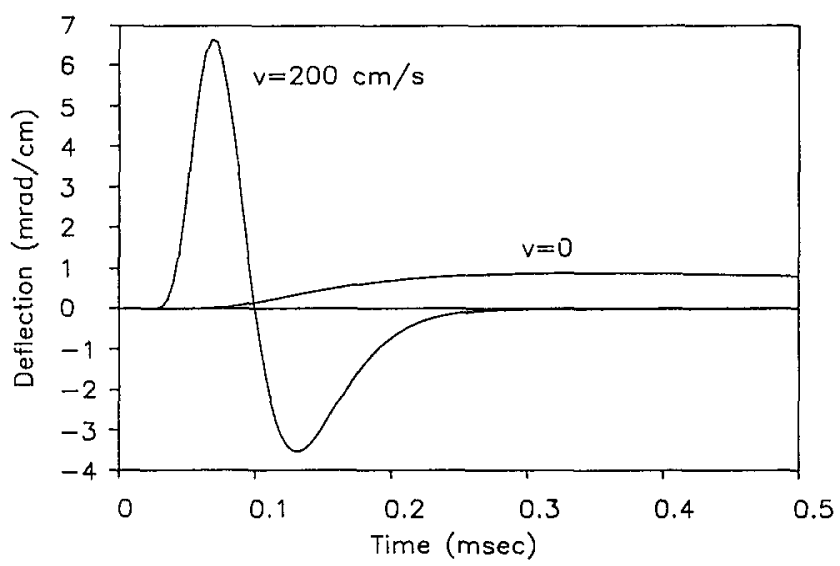

FIG. 7. Beam-deflection signal computed by Eq. (12) for $v=0$ and 200 $\mathrm{cm} / \mathrm{s}$ using $40 \mathrm{~mJ} / \mathrm{cm}^{2}$ with a probe-beam height of $200 \mu \mathrm{m}$. 


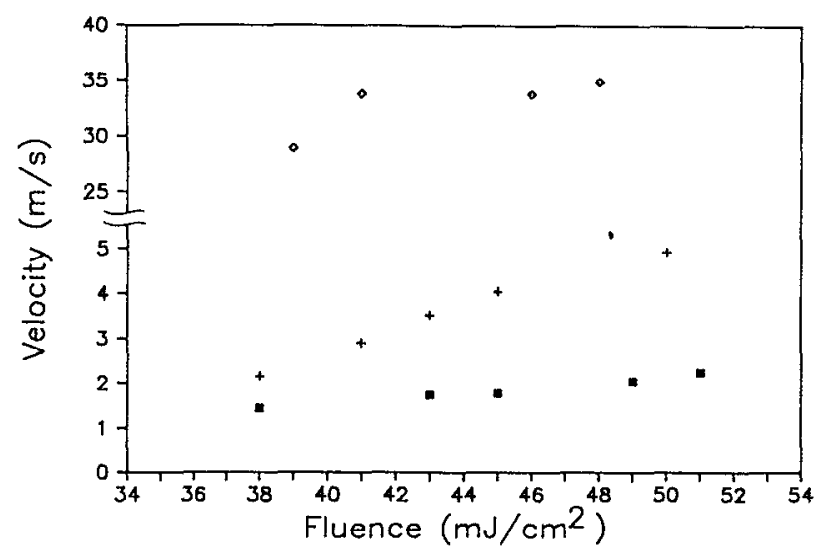

FIG. 8. Gas velocity derived from time-of-flight analysis of the distorted thermal deflection signals for PET in He $(\diamond)$, air $(+)$, and Xe $(\boldsymbol{\square})$ vs laser fluence. Data obtained at a probe-beam height of $300 \mu \mathrm{m}$.

changes. Further modeling would therefore be required to fit the deflection data above the ablation threshold.

Thus, we identify the ablation threshold for PET to be in the range $28-33 \mathrm{~mJ} / \mathrm{cm}^{2}$, which is in good agreement with earlier measurements by other methods $\left(30 \mathrm{~mJ} / \mathrm{cm}^{2}\right.$, Ref. 35, and $22 \mathrm{~mJ} / \mathrm{cm}^{2}$, Refs. 36-38).

The highest-fluence deflection signal shown in Fig. 6 is reminiscent of deflection signals shown in Fig. 3 of Chap. 7 of Ref. 12 where crossed-beam PDS was used to measure gas-flow velocity above a laminar jet. In that work it was shown that the time delay for the deflection signal to cross zero could be used in a time-of-flight analysis to measure flow velocity with good accuracy. Assuming that these distorted deflection signals are due to convection, we applied a time-of-flight analysis to the data in Fig. 6 as well as other data for PET taken at other fluences. The velocity measured by this method is then taken to be the velocity of the air/ablation products mixture normal to the surface. This was done for PET ablation in air, $\mathrm{He}$, and $\mathrm{Xe}$ as a function of excimer-beam fluence at a height of $300 \mu \mathrm{m}$. The measurements were made at fluences from 38 to 51 $\mathrm{mJ} / \mathrm{cm}^{2}$, where it was found (see Fig. 8) that the fluid velocity increased roughly linearly with fluence and ranged from 2.2 to $4.9 \mathrm{~m} / \mathrm{s}$ for air, from 1.4 to $2.3 \mathrm{~m} / \mathrm{s}$ for $\mathrm{Xe}$, and from 29 to $35 \mathrm{~m} / \mathrm{s}$ for He. The velocities measured in air by this method have been confirmed by our schlieren studies. ${ }^{4}$

We have also used this method of measuring the plume velocity at greater probe-sample separations and for a higher fluence shown in Fig. 9. The results are shown for both air and $\mathrm{N}_{2}$, where the gas velocity in $\mathrm{N}_{2}$ is considerably slower than in air. Previously, ${ }^{4}$ we observed that the plume for laser ablation of PET in air was roughly $20 \%$ larger than that in $\mathrm{N}_{2}$. We believe this is due to the presence of oxygen in air, which enables a turbulent diffusion flame to occur for the ablation of PET in air.

It is possible that some of the deflection signal shown in Fig. 6 is due to particulates formed during or slightly after material ablation commences. The particulates would be expected to carry heat and could therefore deflect the

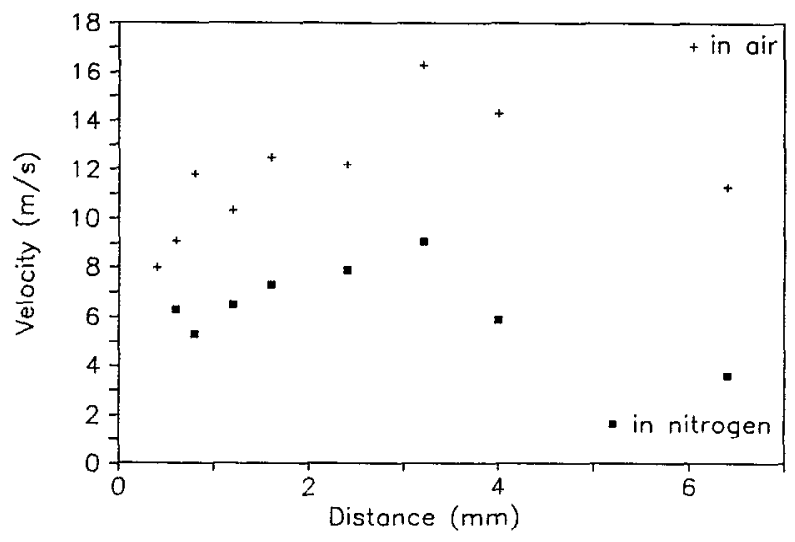

FIG. 9. Gas velocity above PET ablated in air and in $\mathrm{N}_{2}$ as a function of distance above the PET sample. Data obtained at $160 \mathrm{~mJ} / \mathrm{cm}^{2}$.

probe beam. Time-dependent laser-scattering experiments are underway to explore the possibility of particulate ejection.

\section{B. Photoacoustic deflection}

Figure 10 shows a deflection signal obtained at $22 \mathrm{~mJ} /$ $\mathrm{cm}^{2}$ in dry air for PET on a more rapid time scale than above. This two-lobed deflection signal is characteristic of the deflection signal obtained from an acoustic wave. ${ }^{18-24}$ This signal was measured as a function of the probe-sample separation and the time delay until the defiection zero crossing was plotted versus $z$. The slope gave a velocity of $327 \mathrm{~m} / \mathrm{s}$, which is within error bars of the value $330 \mathrm{~m} / \mathrm{s}$ for the speed of sound in air at ambient conditions. Note that the polarity is what one would expect for an acoustic wave; namely, the initial lobe is opposite to that of the thermal signal. This corresponds to a positive density gradient approaching the probe beam followed by a lobe in the opposite direction. We have studied these pulses in $\mathrm{He}$ and

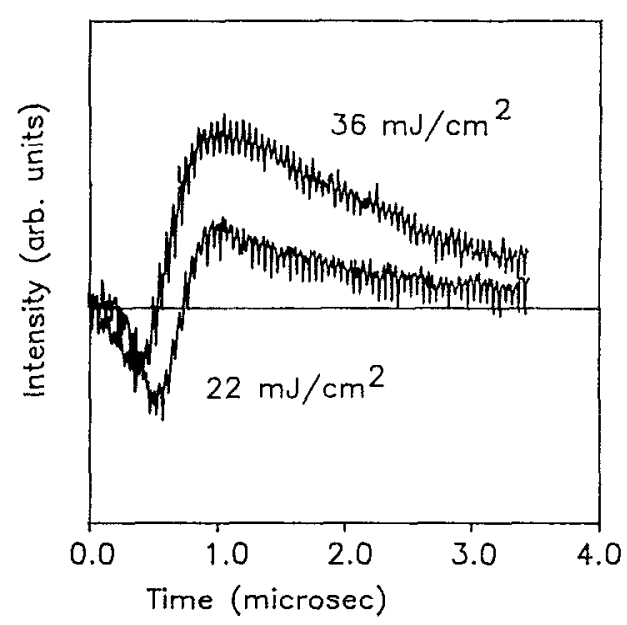

FIG. 10. Acoustic pulse beam-deflection signal for PET in air for two different values of the excimer-beam fluence. Data obtained at a probebeam height of $300 \mu \mathrm{m}$, averaged over 64 shots. 


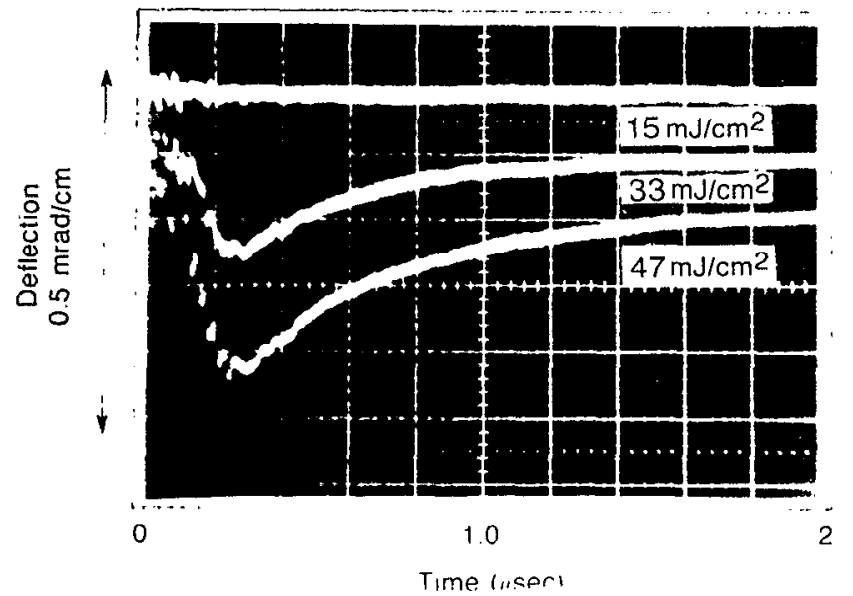

FIG. 11. Beam-deflection signal as a function of fluence for PET obtained in vacuum. Data obtained in a vacuum of $1 \times 10^{-5}$ Torr with a probebeam height of $200 \mu \mathrm{m}$.

obtained the expected acoustic velocity. Figure 10 also shows the distortion of the acoustic pulse at higher fluence. As the fluence is increased to $36 \mathrm{~mJ} / \mathrm{cm}^{2}$, the polarities of the deflection signal are consistent with the evolution of a shock wave from a sound wave. When this occurs one expects the shock's leading edge (negative lobe) to shorten and steepen, while the shock's trailing edge (positive lobe) should broaden. Our schlieren study ${ }^{4}$ indicated a transition from a sound wave below the ablation threshold to a shock wave above threshold. In fact, the shock velocity was found to be linearly dependent on fluence above threshold with a slope of $0.6(\mathrm{~m} / \mathrm{s}) /\left(\mathrm{mJ} / \mathrm{cm}^{2}\right)$ for PET.

In a future paper we will describe measurements of the etch depth by a time-of-flight photoacoustic deflection approach.

\section{Beam deflection in a vacuum}

The defiection signal obtained in a vacuum at very low fluence does not show any deflection at any time. Even at a fluence near or above the ablation threshold, the thermal signal does not appear since there is no fluid to conduct the heat from the sample surface to the probe beam. However, a deflection signal does appear at fast times $(0.2-0.6 \mu \mathrm{s})$ for a fluence above the threshold. Figure 11 shows the deflection signal obtained in a vacuum for three fluences: 15,33 , and $47 \mathrm{~mJ} / \mathrm{cm}^{2}$. The deflection is much more rapid and in the opposite direction to the low-fluence deflection in air. The amplitude of the deflection increases nonlinearly as the fluence is increased through the ablation threshold. A time-of-flight analysis was applied to the vacuum deflection data to obtain ablation product velocities for the samples studied here on the order of $1-3 \mathrm{~km} / \mathrm{s}$. Further analysis of the beam-deflection signal in a vacuum is currently in progress and will be reported later.

\section{CONCLUSIONS}

Photothermal and photoacoustic deflection of a probelaser beam were applied to laser ablation of a polymer. At low fluence the thermal deflection signal can be accounted for quantitatively and allowed new methods of measuring the thermal diffusivity of the air above the sample. A distortion of the photothermal signal was observed as the fluence is raised through the ablation threshold. The velocity of the ablation product/gas mixture was measured as a function of laser fluence and distance from the surface. The photoacoustic beam-deflection signal changed from a sound to a shock wave above the ablation threshold. Beam deflection in a vacuum was used in a time-of-flight measurement of ablation product velocities. This work has demonstrated that beam deflection has potential as a useful diagnostic technique for excimer-laser ablation of a polymer. We have also found the approach works as well using other ablating laser wavelengths (193 nm and $1.06 \mu \mathrm{m}$ ) and for a wide variety of materials.

\section{ACKNOWLEDGMENT}

P. V. and R. G. thank the National Science Foundation for support.

'J. A. Sell, D. M. Heffelfinger, P. Ventzek, and R. M. Gilgenbach, Appl. Phys. Lett. 55, 2435 (1989).

2J. A. Sell, D. M. Heffelfinger, P. Ventzek, and R. M. Gilgenbach in Photoacoustic and Photothermal Phenomena II: Proceedings of the 6th International Topical Meeting, Baltimore, MD, edited by J. C. Murphy, J. W. Maclachlan Spicer, L. C. Aamodt, and B. S. H. Royce, Vol. 62 of Springer Series in Optical Sciences (Springer, Berlin, 1990), p. 194.

${ }^{3}$ P. L. G. Ventzek, R. M. Gilgenbach, J. $\Lambda$. Sell, and D. M. Heffelfinger, Bull. Am. Phys. Soc. 34, 1919 (1989).

${ }^{4}$ P. L. G. Ventzek, R. M. Gilgenbach, J. A. Sell, and D. M. Heffelfinger, J. Appl. Phys. 68, 965 (1990).

${ }^{5}$ W. B. Jackson, N. M. Amer, A. C. Boccara, and D. Fournier, Appl. Opt. 20, 1333 (1981).

${ }^{6}$ A. C. Boccara, D. Fournier, W. Jackson, and N. M. Amer, Opt. Lett. 5, 377 (1980).

${ }^{7}$ J. C. Murphy and L. C. Aamodt, J. Appl. Phys. 51, 4581 (1980).

${ }^{8}$ A. Mandelis, J. Appl. Phys. 54, 3404 (1983).

${ }^{9}$ R. M. Banish, R.-F. Xiao, and F. Rosenberger, J. Appl. Phys. 64, 2907 (1988).

${ }^{10} \mathrm{H}$. Sontag and A. C. Tam, Opt. Lett. 10, 436 (1985).

11 J. A. Sell, Appl. Opt. 24, 3725 (1985).

${ }^{12}$ J. A. Sell, Photothermal Investigations of Solids and Fluids, edited by $\mathbf{J}$. A. Sell (Academic, Boston, 1989).

${ }^{13}$ H. Sontag, A. C. Tam, and P. Hess, J. Chem. Phys. 86, 3950 (1987).

${ }^{14}$ F. K. Fotiou and M. D. Morris, Appl. Spectrose, 40, 700 (1986).

${ }^{15}$ A. C. Tam and H. Schroeder, J. Appl. Phys. 64, 3667 (1988).

${ }^{16}$ J. C. Loulergue and A. C. Tam, Appl. Phys. Lett. 46, 457 (1985).

${ }^{17}$ S. I. Yun, K-D. Oh, K-S. Ryu, C-G. Kim, H. L. Park, H. J. Seo, and C. Kum, Appl. Phys. B 40, 95 (1986).

${ }^{18}$ A. C. Tam, W. Zapka, H. Coufal, and B. Sullivan, J. Phys. (Paris) Colloq. 44, C6-203 (1983).

${ }^{19}$ W. Zapka, P. Pokrowsky, and A. C. Tam, Opt. Lett. 7, 477 (1982).

${ }^{20}$ W. Zapka and A. C. Tam, Appl. Phys. Lett. 40, 310 (1982).

${ }^{21}$ A. C. Tam and W. P. Leung, Phys. Rev. Lett. 53, 560 (1984).

${ }^{22}$ A. C. Tam, Rev. Mod. Phys. 58, 381 (1986).

${ }^{2.3}$ A. Rose, G. J. Salamo, and R. Gupta, Appl. Opt, 23, 781 (1984).

${ }^{24}$ A. Rose and R. Gupta, Opt. Commun. 56, 303 (1986).

${ }^{25}$ G. C. Wetsel, Jr., S. A. Stotts, and C. G. Clark, J. Phys. (Paris) Colloq. 44. C6-67 (1983).

${ }^{26}$ G. E. Jamieson and G. C. Wetsel, in IEEE 1985 Ultrasonics Symposium Proceedings, San Francisco, CA, 1985, edited by B. R. McA voy (IEEE, New York, 1985), p. 451.

${ }^{27}$ G. Koren, Appl. Phys. Lett. 51, 569 (1987).

${ }^{28}$ S. Petzoldt, A. P. Elg, M. Reichling, J. Reif, and E. Matthias, Appl. Phys. Lett. 53, 2005 (1988).

${ }^{29}$ J. Reif, S. Petzoldt, A. P. Elg, and E. Matthias, Appl. Phys. A 49, 199 (1989). 
${ }^{30}$ C. L. Enloe, R. M. Gilgenbach and J. S. Meachum, Rev. Sci. Instrum. 58, 1597 (1987).

${ }^{31}$ C. L. Enloe, Ph.D. thesis, Department of Nuclear Engineering, University of Michigan, 1988.

${ }^{32}$ R. W. Dreyfus, F. A. McDonald, and R. J. von Gutfield, J. Vac. Sci. Technol. B 5, 1521 (1987).

${ }^{33}$ R. W. Dreyfus, F. A. McDonald, and R. J. von Gutfield, Appl. Phys. Lett. 50, 1491 (1987).
${ }^{34}$ See Sec. 14.6.9 of H. S. Carslaw and J. C. Jaeger, Conduction of Heat in Solids, 2nd ed. (Oxford Science, Oxford, 1986).

${ }^{35}$ P. E. Dyer and J. Sidhu, J. Appl. Phys. 57, 1420 (1985).

${ }^{36}$ S. Lazare and V. Granier, J. Appl. Phys. 63, 2110 (1988).

${ }^{37}$ S. Lazare and V. Granier, Laser Chem. 10, 25 (1989).

${ }^{38}$ S. Lazare, J. C. Soulignac, and P. Fragnaud, Appl. Phys. Lett. 50, 624 (1987). 